\title{
Transit, Astrometric, Coronagraphic and Interferometric Exo-planet Studies - Synergy and Complementarity
}

\author{
W. A. Traub ${ }^{1}$, S. T. Ridgway ${ }^{2}$, C. A. Beichman ${ }^{3}$, \\ K. J. Johnston ${ }^{4}$, J. Kasting ${ }^{5}$, and M. Shao ${ }^{6}$ \\ ${ }^{1}$ Jet Propulsion Lab, M/S 301-451, 4800 Oak Grove Dr., Pasadena, CA, 91109, USA \\ email: wtraub@jpl.nasa.gov \\ ${ }^{2}$ NASA Headquarters, 300 E St SW, Washington, DC 20546 \\ email: sridgway@hq.nasa.gov \\ ${ }^{3}$ Michelson Science Center, California Inst. of Technology, Mail Code 100-22, 770 South \\ Wilson Avenue, Pasadena, CA 91125, USA \\ email: chas@ipac.caltech.edu \\ ${ }^{4}$ U.S. Naval Observatory, 3450 Massachusetts Ave, NW, Washington DC 20392, USA \\ email: kjj@astro.usno.navy.mil \\ ${ }^{5}$ Department of Geosciences, 443 Deike, Penn State University, University Park, PA 16802, \\ USA \\ email: kasting@essc.psu.edu \\ ${ }^{6}$ Jet Propulsion Lab, M/S 301-486, 4800 Oak Grove Dr., Pasadena, CA, 91109, USA \\ email: mshao@huey.jpl.nasa.gov
}

\begin{abstract}
The goals of the Navigator Program at NASA are to find Earth-like planets around nearby stars, to determine if they are habitable, and to search for signs of life. Three strategic missions are planned to carry out this program: the Space Interferometer Mission Planetquest (SIM), the Terrestrial Planet Finder Coronagraph (TPF-C), and the Terrestrial Planet Finder Interferometer (TPF-I). These missions, along with the PI-class Kepler project, will each discover unique knowledge about extrasolar planets, synergistically building on the other missions.
\end{abstract}

Keywords. techniques: high angular resolution, interferometric; stars: planetary systems.

\section{Introduction}

Over 169 extrasolar planets have been discovered since 1996. These planets were detected using Doppler spectroscopy instruments that are sensitive to large, massive objects like our Solar System's giant planets. Other types of instruments will be needed to detect "terrestrial planets" like Venus, Earth, and Mars. NASA $\dagger$ and the US astronomy community currently plan four distinct missions to search for terrestrial planets. This set of missions is designed to return maximum scientific benefit, and in particular to tell us, beyond reasonable doubt, if nearby planets can be found that are habitable and show signs of life. These missions have a synergistic relationship - while each can stand alone, the scientific impact will be reduced substantially if fewer missions are launched.

$\dagger$ For simplicity, the following analysis refers to the current NASA suite of planned major exo-planet missions. This does not exclude other possible NASA missions, or changes in scope of those discussed. Of course, a variety of other missions (COROT, GAIA, DARWIN) capture some or even considerable elements of this science - NASA and ESA consider that DARWIN and TPF-I could be a single joint mission. 


\section{Missions to Detect and Characterize Extrasolar Planets}

At the highest level, the logical relation among the methods of exo-planet detection and the study of Earth-like planets can be understood as follows. Radial velocity programs show, by detection of many planets, that there is indeed a science of exo-planets. Transit detections of large numbers of low-mass planets (Kepler) will show that there is indeed a science of exo-Earths (though the detected objects will be too distant for detailed study). SIM will survey nearby systems, and find exo-Earths that can be studied in detail. TPF-C and TPF-I will show that exo-Earth habitability can be studied.

Kepler will detect planets indirectly by measuring the dimming of a star when a planet transits in front of the stellar disk. Kepler will tell us the size of the planet, how close it is to the star, and the length of its year, using the measured depth, duration, and repeatability of dimming. It will do this with a single small telescope, staring at a distant field of stars (not nearby stars) for several years. Kepler will detect up to hundreds of terrestrial planets in the habitable zone, as well as determining the albedo, size, mass, and density (the latter two when augmented with planned RV data) of short period giant planets. Kepler will tell us for the first time whether terrestrial planets are common or rare - something about which we can only guess today.

SIM uses interferometers to measure stellar positions with respect to neighboring stars, and repeats these measurements over the full sky, many times over several years, to detect planets indirectly by measuring the astrometric wobble of a star. SIM will tell us the mass of each planet, its orbital distance, its orbital shape and orientation, and the length of its year. SIM will be most sensitive to planets that have periods of up to several years. SIM will tell us which nearby stars have such planets, as well as the relative numbers of heavy- and light-weight planets, down to Earth-mass range around some stars. If Kepler finds that Earths are plentiful, SIM might search a smaller number of systems to a deeper limit, while if Earths are rare, SIM might conduct a larger, shallower survey. SIM could search for gas giants in systems for which Kepler finds terrestrial planets.

TPF-C uses a single, large-aperture telescope and a coronagraph to suppress starlight. It will detect and characterize planets directly with a visible-wavelength snapshot image of a star-planet system. TPF-C will be able to tell us the visible brightness of each planet, its orbital distance, its orbital shape and orientation, the length of its year, and the amounts of life-related atmospheric gases (oxygen, water vapor, ozone, carbon dioxide, methane). In favorable cases, TPF-C potentially will be able to tell us the length of the planet's day, the amount of plant life on the surface, an estimate of ocean/land ratio, and a measure of cloud variability. TPF-C will be most sensitive to Earth-like planets in the habitable zone that are in orbital positions such that their day-lit portions are visible from the Earth. TPF-C will be able to observe planets that are, on average, smaller than the planets that Kepler, SIM, and radial-velocity techniques can reveal. TPF-C will be designed to search for habitability and signs of life on extrasolar planets.

TPF-I is the infrared analog to TPF-C and uses an array of four formation-flying large, cooled telescopes with interferometric nulling beam combination to suppress the star's emission. TPF-I will detect and characterize planets directly, using the planet's own infrared emission, independent of the orientation of the day-lit side. TPF-I will be able to tell us the infrared brightness of each planet, its orbital distance, its orbital shape and orientation, the length of its year, the amounts of life-related atmospheric gases (water vapor, ozone, carbon dioxide, methane, and nitrous oxide). If the planet's atmosphere is transparent enough, TPF-I may be able to tell us the length of the planet's day, an estimate of ocean/land ratio, and a measure of cloud variability. Together, TPF-C and TPFI are an ideal pair to search for habitability and signs of life on extrasolar planets. 


\section{Mission Connections and Synergy}

The four missions, Kepler, SIM, TPF-C, and TPF-I, approach the search for terrestrial planets from different perspectives, and all perspectives are needed to establish mission requirements, to determine habitability and to search for signs of life. The rest of this discussion focusses on the missions which conduct detailed study of nearby exo-planets SIM, TPF-C, and TPF-I.

\subsection{Stable orbit in habitable zone}

Each mission can measure an orbit and determine if it lies within the habitable zone (where the temperature may permit liquid water on the surface of the planet).

(1) A SIM detection of a terrestrial-mass planet could provide each TPF with targets to be characterized and the optimum times for observing them, thus increasing the TPF early-mission characterization yield.

(2) Where SIM finds a planet, of any mass, in almost any orbit, TPF-C and TPF-I will want to search as well, because we expect that planetary multiplicity may well be the rule (as in our Solar System).

(3) For cases where SIM detects a planet marginally, a TPF can verify or reject the detection. Such verifications can mutually enhance the significance of results from SIM and TPFs, no matter in what order the missions are carried out.

\subsection{Planet temperature and temperature variability due to distance changes}

A planet's effective temperature can be roughly estimated by noting its distance from its star, and by assuming a value for the albedo. TPF-I can observe directly the thermal infrared emission continuum at several wavelengths (i.e., infrared color), and use Planck's law to calculate the effective temperature. For a planet with a thick or cloudy atmosphere (like Venus), the surface temperature is different from the effective temperature, but might be inferred from a model of the atmosphere. With all three missions combined, the orbit, albedo, and greenhouse effect can be estimated, and the surface temperature as well as temperature fall-off with altitude can be determined cooperatively and more accurately than with any one mission alone.

Each mission alone can observe the degree to which the orbit is circular or elliptical, and thereby determine if the temperature is constant or varying. The measurement of a terrestrial planet's orbital eccentricity using combined missions (SIM plus TPF) can be much more accurate than from any one mission alone, because complementary sensitivity ranges in planet mass and distance from star combine favorably.

\subsection{Planet radius and albedo}

Planet radius and mass, or equivalently density, are very important for determining the type of planet (rocks, gas, ice, or combination), its habitability (solid surface or not; plate tectonics likely or not), and its history (formed inside or outside of ice line). SIM measures planet mass, from which we can estimate radius to within a factor of 2 (assuming a value of density, which in the Solar System spans a factor of 8). TPF-C measures visible brightness, which along with an estimate of albedo, can give a similarly rough estimate of radius. TPF-I measures infrared brightness and color temperature, which with Planck's law gives a more accurate planet radius. With SIM's mass, and one or both TPF brightness measurements, we can dramatically improve the estimate of planet radius.

The albedo is important because it controls the planet's effective temperature, which is related to its habitability. SIM and TPF-C combined can estimate possible pairs of values of radius and albedo, but cannot pick which pair is best. We can make a reasonable 
estimate of albedo by using TPF-C to measure the planet's color, then appealing to the planets in our Solar System to convert a color to an absolute albedo. By adding TPFI measurements we can determine radius (above), then with brightness from TPF-C we can compute an accurate albedo, and therefore effective temperature and potential habitability.

\subsection{Planet mass and surface gravity}

SIM measures planet mass directly. TPF-C and TPF-I depend on SIM for planet mass. If TPF-C and TPF-I do not have a SIM value for planet mass, then they will use theory and Solar System examples to estimate masses. SIM plus TPF-C and TPF-I are needed to distinguish among rock-, ice-, and gas-dominated planet models, and to determine with confidence whether the planet could be habitable. SIM will typically be able to find nearby heavy-Earth planets, pointing the way for TPF to find Earth-mass and Mars-mass planets. We can understand these mass values qualitatively, as follows. SIM can most easily detect a stellar wobble when the planet is heavy, and the instrument's astrometric threshold is sufficiently small. TPF-C can most easily detect a planet when it has a large reflecting surface area per unit mass (i.e., smaller diameters), and the instrument contrast threshold is sufficiently small. TPF-I can have a smaller inner working angle than TPF-C, and so could see planets closer to the parent star. Several synergies result.

(1) TPF might initially avoid a case for which SIM finds a massive planet in an orbit that would make it impossible for a terrestrial planet to exist in the habitable zone.

(2) Since SIM can survey many stars relatively quickly, it can produce a list of stars where habitable-zone planets are strongly or even marginally detected, and hand off this list to TPF for confirmation, and characterization, thereby increasing the early-mission efficiency of the TPF missions.

(3) For SIM detections, TPF-C and TPF-I can confirm the detection and measure the planet's colors, to build up a catalog of extrasolar planet properties.

(4) For SIM detections that have low signal-to-noise ratios, TPF-C and TPF-I can improve the orbit parameters.

Surface gravity is important to habitability because massive, dense planets are more likely to have plate tectonics (a crucial factor in Earth's evolution), and retain an atmosphere (also crucial for Earth). The planet's surface gravity is calculated directly using mass from SIM and radius from TPF-C and TPF-I. Cooperative measurements are the only way to obtain this data.

\subsection{Atmosphere and surface composition}

The TPF missions are designed to measure a planet's color and spectra, from which we can determine the composition of the atmosphere and surface. For the atmosphere, TPF$\mathrm{C}$ can measure water, molecular oxygen, ozone, the presence of clouds for a planet like the present Earth, and in addition it can measure carbon dioxide and methane for a planet like the early Earth or a giant planet. For the surface TPF-C can measure vegetation using the "red edge" effect. TPF-I will add to this suite of observations by measuring carbon dioxide, ozone, water, methane, and nitrous oxide using different spectroscopic features, and in general probing a different altitude range in the atmosphere. SIM is important to this interpretation because it provides planet mass, crucial to interpreting atmospheric measurements.

Both TPF-C and TPF-I are needed in order to determine whether a planet is habitable, because they make complementary observations, as follows (assuming an Earth-like planet). Ozone has a very strong infrared (TPF-I) feature, and a weak visible (TPF-C) one, so if ozone is abundant, both can be used to extract the abundance as well as the 
thermal structure of the atmosphere - if ozone is weak, only the TPF-I feature will be useable. Water as seen by TPF-C will be near the surface of the planet, but as seen by TPF-I it will be in the upper atmosphere; together both give a more complete picture of the atmosphere. Methane, carbon dioxide and nitrous oxide, in small amounts (as for the present-day Earth) can be detected by TPF-I. Methane and carbon dioxide, in large amounts (as for the early Earth), can be detected by TPF-C. For large amounts of methane or carbon dioxide, TPF-I will see mainly the amount in the upper atmosphere, but TPF-C will see mainly the amount in the lower atmosphere - both are needed for a complete picture. In addition to these overlap topics, only TPF-C can measure oxygen, vegetation, and the total column of air (Rayleigh scattering); likewise only TPF-I can measure the effective temperature.

Both TPF-C and TPF-I have water absorption features in their spectra, so if water vapor is present in the atmosphere, we will be able to measure it. However habitability requires liquid water on the surface, which in turn requires a solid surface as well as a temperature that permits the liquid state; only with the help of a value of mass from SIM will we be able to know the radius, and when TPF-I is launched, the temperature. To know whether liquid water is present on the surface of a planet, we need mass data from SIM, and spectroscopic water data from TPF-C or TPF-I.

Both TPF-C and TPF-I potentially can measure changes in color and the strengths of spectral features as the planet rotates. These changes can tell us the length of day on the planet, and can indicate the presence of large oceans or land masses (with different reflectivities or emissivities). Superposed on this time series of data could be random changes from weather patterns, possibly allowing the degree of variability of weather to be measured. The TPF missions can potentially measure variability of composition over time, which we know from our Earth to be an indicator of habitability.

\subsection{Other planets, comets, asteroids, and zodiacal dust}

All three missions can detect several planets around a star, within their ranges of sensitivity. Thus there may be a planet close to the star that SIM can detect, but is hidden from TPF-C. Likewise there may be a distant planet that TPF-C or TPF-I can detect, but has a period that is too long for SIM. For the more subtle issue of whether the planets have orbits in or out of the same plane, SIM will do the best job. In general, each of the three missions will detect some but not necessarily all of the planets that might be present in a system, so the combination will deliver a complete picture of what planets are present, their masses, their orbits, and how they are likely to influence each other over the age of the system, including co-planarity.

Comets and asteroids have very low masses compared to planets, so will not be detected by SIM, but because they have a large surface area (for their mass) they might be seen collectively, if their numbers are high, by TPF-C in reflected light and TPF-I in thermal emission. The combination of TPF-C (visible) and TPF-I (infrared) measurements can tell us about the average albedo and numbers of solid objects (comets and asteroids), as well as ground-up material (exo-zodiacal dust). The detection of sub-planet material will produce a picture of a planetary system's history and present interactions.

\subsection{Atmospheric and surface biomarkers}

The simultaneous presence of an oxidized species (like oxygen or ozone) and a reduced species (like methane) is considered to be a sign of non-equilibrium that can indicate indirectly the presence of life on a planet. The presence of a large amount of molecular oxygen, as on the present Earth, may also be an indirect sign of life. Since water is a prerequisite for life, as we consider it here, the presence of liquid water (indicated by 
water vapor and an appropriate temperature) is needed. Together these spectroscopicallydetectable species are our best current set of indicators of life on a planet. These markers will be measured exclusively by TPF-C and TPF-I, but to know that we are observing an Earth-like planet will require SIM data on mass.

The red edge is a property of land plants and trees whereby they are very good reflectors of red light (just beyond the long-wavelength limit of our eyes). This is a useful feature for measuring plant cover on Earth. If extrasolar planets have developed plant life like that on Earth, and if the planet is bright, has few clouds, and a lot of vegetated land area, then we may use this feature to detect living vegetation.

\subsection{Beyond exo-planet studies - general astrophysics and technology}

Each mission, SIM, TPF-C, and TPF-I, offers a uniquely new and powerful capability in angular resolution, in deep and high contrast imaging, or in both. Vigorous programs of general astrophysics are expected to develop their own synergies between these missions and other observational platforms. Examples include stellar evolution (from star forming regions to late stars), cosmology (improved measurements of $\mathrm{H}_{0}$ ), study of dark matter in the local galaxy group and beyond, and massive compact objects (by enabling microlensing studies from widely separated stations).

Each new mission builds on the technological heritage of earlier related missions, and in some cases that inheritance is essential. SIM has developed exquisitely precise methods of measuring the relative locations of optical elements in a train of optics and of accounting for wavefront uncertainties in optical systems, a technological heritage that will be beneficial to the TPFs. SIM will be the first large interferometer flown in space, and will be the first space instrument to demonstrate aperture synthesis imaging, paving the way for the next generation of dilute-aperture missions, including TPF-I.

\section{Conclusions}

The exploration of extrasolar planetary systems is a rich and diverse field. It calls for measurements with many kinds of instruments, as well as theoretical studies and numerical modeling. To discover and characterize extrasolar planets that are habitable and may show signs of life, and to be sure beyond a reasonable doubt that we can detect life, we need to measure the statistical distribution of planet diameters, the masses of nearby planets, and the spectra at visible and infrared wavelengths. Kepler, SIM, TPF-C, and TPF-I can carry out these measurements. In short, Kepler is needed for statistical information on the existence of terrestrial planets. SIM is needed for identification of nearby target systems and planets and measurement of planet masses. TFP-C and TPFI are needed to evaluate the atmospheres for habitability. All are needed to characterize exo-planet systems. Not only does each mission provide its own compelling science, but together these missions form a coherent approach that will advance our understanding better than any single one by itself.

\section{Acknowledgements}

We are grateful to the members of the SIM Science Team, the TPF-C Science and Technology Definition Team, the TPF-I Science Working Group, and to Kepler PI William Borucki, for their contributions to this study. 